\title{
Study on Storable Down-hole Drilling String Vibration Testing System
}

\author{
Liang $\mathrm{Ge}^{1,2, *}$, Pan $\mathrm{Hu}^{1}, \mathrm{Ze} \mathrm{Hu}^{3}$, Pin Chen ${ }^{4}$, Qing Yang ${ }^{1}$, Zhengyin Wang ${ }^{1}$, Junbi Liao ${ }^{2}$ \\ ${ }^{I}$ College of Mechanical and Electronic Engineering, Southwest Petroleum University, Chengdu, China, 610500 \\ ${ }^{2}$ Department of Measuring and Control, Sichuan University, Chengdu, China, 610500 \\ ${ }^{3}$ College of Electric and Information, Southwest Petroleum University, Chengdu, China, 610500 \\ ${ }^{4}$ State Key Laboratory of Oil \& Gas Geology and Exploration, Chengdu, China, 610500
}

\begin{abstract}
Drilling engineering is a high input and risk underground project, which needs the real-time down-hole diagnosis and forecast technique. Drilling string vibration signal contains full and complex vibration waves, which contains the vibration of the drilling string caused by itself, the vibration stimulated by the interaction of bit and the formation, the vibration induced by colliding between drill string and bore hole. By adopting drilling string vibration testing system, downhole vibration can be measured in order to grasp and analyze the state of down-hole. Drilling string vibration testing system adopts the ADuC841 MCU as the core chip, and uses the high reliability technology in the design of the software and hardware. The storable down-hole drilling string vibration testing system has advantages of small size, low power consumption, vibration resistance and high temperature resistance. By high temperature test, vibration test, laboratory simulation test and field test, it proves that the down-hole drilling string vibration testing system has the ability to accurately record the actual down-hole drill string dynamic vibration status, and the system can meet the needs of the drilling site testing.
\end{abstract}

Keywords: ADuC841, data acquisition, drilling string, drilling engineering, vibration testing.

\section{INTRODUCTION}

Drilling plays a major role in the development of petroleum and oil field exploration. It masters the various formation parameters on time and monitors the conditions underground while drilling. Traditionally, the field technicians distinguish abnormity and diagnose fault according to the trend of drilling parameters and their experience. Now, the prediction and analysis of underground conditions mostly depend on measuring and analyzing the abnormal changes of drilling engineering parameters [1]. The drill string vibration signal is a comprehensive vibration signal which mainly includes the drill string vibration, the drill bit vibration while breaking formation and the vibration produced by the friction between the drill string and shaft lining. This signal reflexes the working condition of drill string and thus can be an essential basis of the prediction and analysis of underground conditions.

Currently, the underground vibration test system mainly adopts underground measurement while drilling (MWD) and ground measurement [2]. MWD technology requires people to install the sensors near drill bit and then it sends the drill string vibration signal to ground computers for data analysis by wireless or wired transmission. MWD has the characteristic of strong real-time, but it requires the test system to work in the high temperature, high pressure, strong vibration and corrosion environment. Besides, since this way has high

*Address correspondence to this author at Southwest Petroleum University, Chengdu, 610500, P.R. China; Tel: 13980784786;

E-mail: cgroad@163.com requirement of data transmission system and causes high cost, therefore, it hasn't been used widely [3]. As for ground measurement, people need to install the vibration test sensors on the drill string and analyze the vibration condition of underground drill string by measuring the vibration signals on the ground. Although this way reduces the complexity of the environment, yet the testing data cannot reflex the really underground conditions while drilling. Considering these deficiencies of the two measuring methods, a storable downhole drilling string vibration testing system has been developed to extract down-hole drilling information in real-time, avoid the accidents of drilling tools and abnormal drilling situations caused by vibration effectively, shorten the drilling period and save cost.

\section{RULE ABOUT DRILL STRING VIBRATION}

\subsection{Rule of the Drill String Longitudinal Vibration}

While drilling, the unevenness of bottom hole, the contact between teeth interval of cone bit and bottom hole, and the unstable reaction while drill bit breaking rocks, can exert exciting force over drilling tool which causes longitudinal vibration. A placid longitudinal vibration which has small amplitude and vibration frequency is the vertical vibration caused by the contact between bottom hole and drill bit. But when the frequency of the exciting source approaches to any rank innate frequency of drill string, the drill tool will produce resonance, which will make the drill bit divorced from the bottom hole at a moment then bouncing in it. 


\subsection{Rule of the Drill String Lateral Vibration}

The drill string lateral vibration which has an effect on the drill string movement is an important form of the drill string vibration, which can also be called "string vibration". The influencing factors of the lateral vibration while drilling are the fossa dynamic which is caused by the underground drill tool, the axial alternating force and displacement of drill bit. The shaft lining and drill string construction makes a great influence on the drill string lateral vibration.

\subsection{Rule of the Drill String Torsional Vibration}

The friction and collision between the drill string and shaft lining always exist while drilling, because of the bending of the drill string and the irregularity of shaft lining, and the incessant periodic change of reaction power size while the drill string bit breaking the rocks usually leads to the drill tool torsional vibration.

\section{SYSTEM FRAMEWORK DESIGN}

\subsection{Technical Index}

The design objective is to research on a storable downhole drilling string vibration testing system. By this system, the field technicians can comprehend and analyze the underground condition according to the underground vibration signals. And it can also help to provide accurate field data and scientific reference for the oilfield development and direct drilling [4]. The functions of the system include the vibration signals detection, data acquisition, data storage and data playback, and are implemented in the multi-function circuit board. The circuit board is fixed in a groove, which is used to measure the mechanical short section. Considering the design environment of the underground components (including the mechanical components, electronic devices and sensors), the fixation needs to match the following environmental conditions: at the maximum pressure to be $100 \mathrm{MPa}$, the maximum temperature is $125^{\circ} \mathrm{C}$. And the technical indexes are: the implementation of the longitudinal, lateral and torsional vibration; the measuring range of the vibration acceleration is $0-10 \mathrm{~g}$; the resolution is $0.01 \mathrm{~g}$; the storage capacity is $64 \mathrm{MB}$, and the longest continuous working time is 3 days.

\subsection{Circuit System Framework Design}

The storable drilling string vibration testing system can be regarded as a high reliable intelligent system for the underground parameters acquisition and storage. This system is composed of testing subsystem and playback subsystem. The testing subsystem is used for parameters signal detection, signal condition, data acquisition and storage. And the playback subsystem is used for data playback, data analysis and treatment. The whole system must work in the coordinate and cooperation of the two systems and its whole design scheme is as shown in Fig. (1).

Functions of each part:

- Axial accelerometer circuit: detecting acceleration signal.
- Signal processing circuit: processing which includes amplifying and filtering the signals output from axial accelerometer.

- Microcontroller circuit: collecting and controlling the acceleration data through MCU ADuC841.

- Data memory circuit: storing the acceleration data.

- System power circuit: providing $3 \mathrm{~V}$ system power for the system circuit and sensors.

- PC: processing replayed data and analyzing feature.

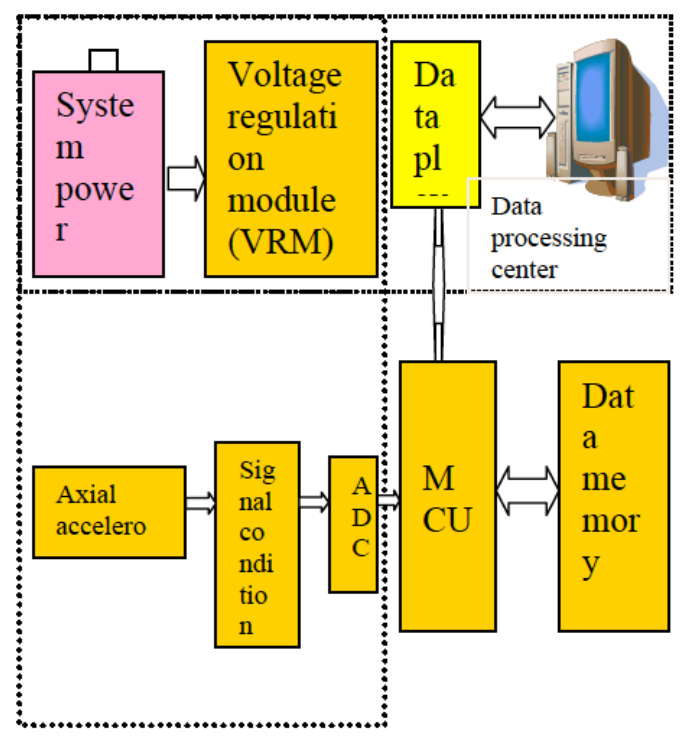

Fig. (1). Block diagram of system.

\section{HARDWARE OF SYSTEM CIRCUIT DESIGN}

\subsection{Sensor Selection}

Because the underground vibration sensor works in thousands of meters underground environment, it needs great stability and temperature resistance and must be selected strictly. The volume, linear range, frequency response characteristic, measuring range, signal outputting method, sensitivity and precision of the sensor must be considered carefully [5]. Compared with the vibration sensors in the available at the market presently, and in order to realize the longitudinal, lateral and torsional vibration testing, this design adopts the new three-axis piezoelectric accelerometer and installs it in the mechanical short section by thread.

\subsection{Signal Processing Circuit Design}

In the design, the charge amplifier is used as a signal condition because the sensor output the charge signal. It is not to amplify the charge but to convert a high internal resistance charge source into a low internal resistance voltage source. To meet the demand of the full-scale of AD [6], 1$3 \mathrm{~V}$ is the best scope of the charge amplifier output voltage. And in order to guarantee the circuit gain, the feedback capacitance can't be too large. The circuit is as shown in Fig. (2). Considering the output charge of the piezoelectric accelerometer, the feedback capacitance $\mathrm{C}_{\mathrm{f}}$ is always not more than $10000 \mathrm{PF}$. At the same time the feedback capacitance 
also cannot be too small. Because the parasitic capacitance would bring trouble to circuit debug and use, so $\mathrm{C}_{\mathrm{f}}$ should not be less than 100PF.

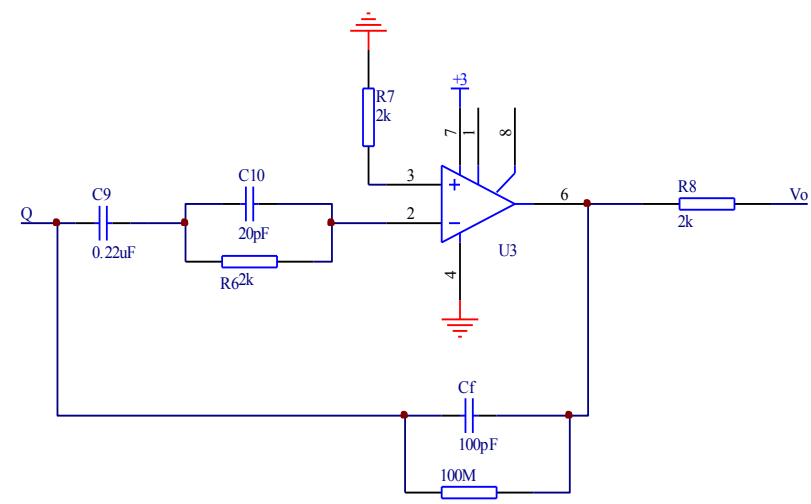

Fig. (2). Circuit of charge amplifier.

\subsection{Data Acquisition and Storage Circuit}

$\mathrm{ADuC841}$ is a newcomer of the high-performance 8-bit microcontroller, and its design is based on the compatible core of 8052 processor. The 12-bit Serial Analog-to-Digital Converter is used to meet the acquisition of acceleration resolution and the memory chip AT45DB321D is used to store data. The data storage circuit is as shown in Fig. (3).

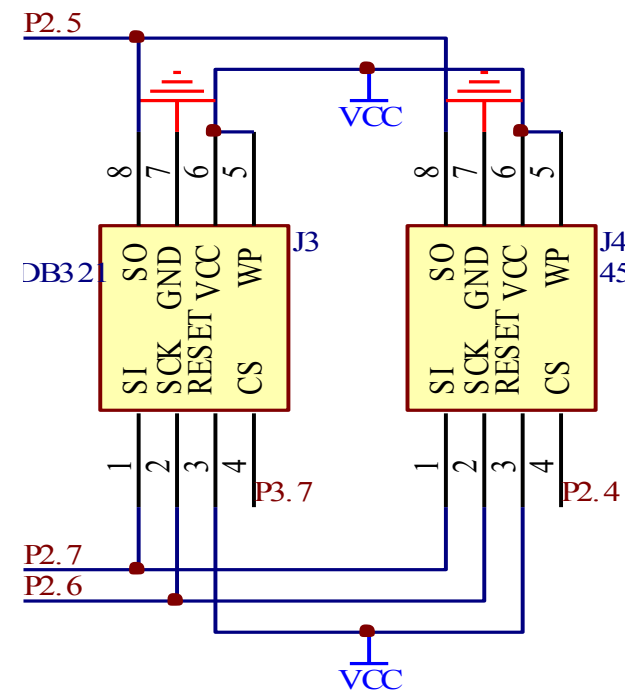

Fig. (3). Circuit of data storage.

\subsection{Data Playback Circuit}

To accomplish data playback, the storable down-hole drilling string vibration testing system transmits vibration data tested by system serial communication circuit to ground computer through the memorizer [7]. Under the condition of keeping up the output level of RS-232 protocol, the serial communication chip MAX3223 adopted by this system will reach the data transmission rate of $115200 \mathrm{bps}$.

\subsection{System Power}

In recent years, with the rapid expansion of portable digital products, battery technology is also developed rapidly.
Considering the system's working environment, the battery of the storable down-hole drilling string vibration testing system must be supplied by temperature lithium battery. To meet the demand of system circuit, the $3.6 \mathrm{~V}$ output of temperature lithium battery must be converted into $3 \mathrm{~V}$ through the power conversion chip.

\subsection{System Software Design}

The system software design is divided into testing subsystem software design and playback subsystem software design. The testing system software is used to undertake microcontroller to control ADC for data acquisition and storage, and data transmission. The playback subsystem software accomplishes the task of vibration data playback, analysis, the feature extraction and so on by the virtual instrument software. The soft function structure is as shown in Fig. (4).

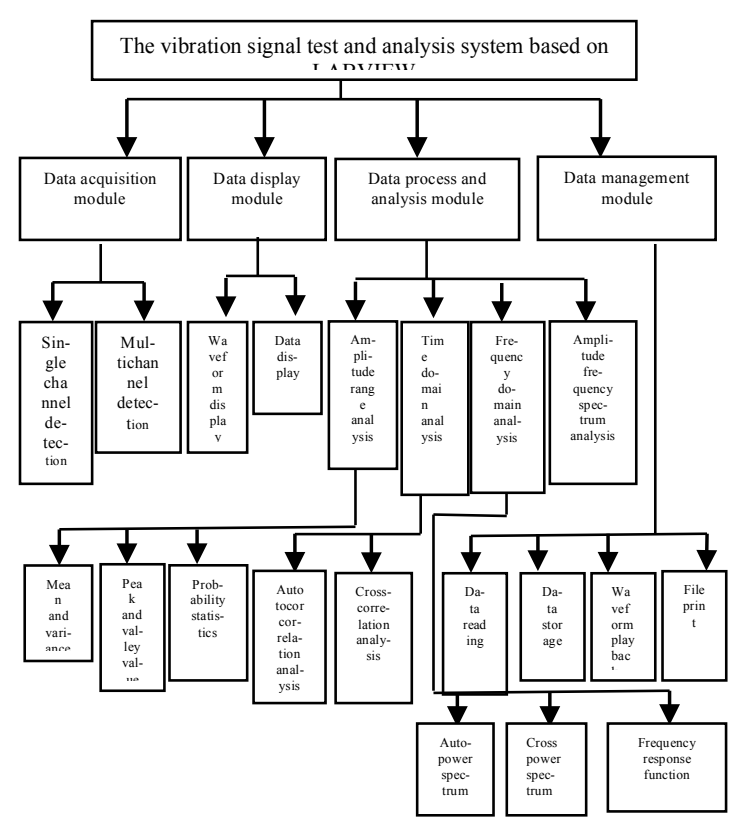

Fig. (4). Function of data playback and process software.

\section{SYSTEM TESTING}

In the hardware and software design process, to ensure that the storable down-hole drilling string vibration testing system works normally, the hardware and software must be tested and debugged [8]. And in order to meet the demand of underground actual testing and use, it is necessary to take the high temperature test, vibration test and laboratory simulation test. Through the high temperature test and vibration test, the instrument used in the underground work environment is simulated to verify the heat resistance and antivibration performance of the system and test the system reliability. And through the laboratory simulation test, the vibration signal input is simulated to verify the accuracy of the system function [9-10].

After the laboratory simulation test, it is followed by the field test. The well depth of system is 1800 meters at which the field data is collected successfully. A group of vibration 
data obtained by the system from smooth drilling is as shown in Fig. (5).

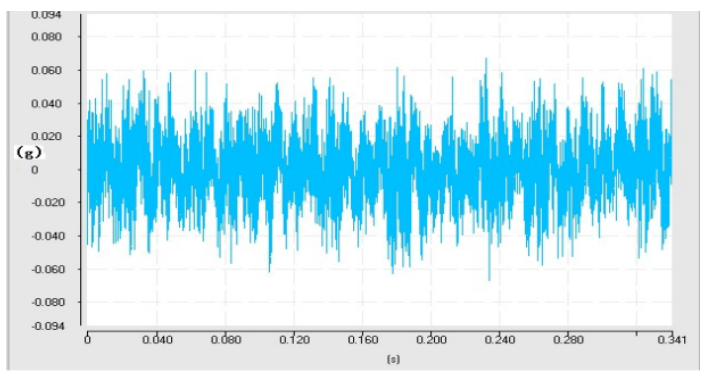

Fig. (5). Down-hole longitudinal vibration signal.

By comparing the test data and field conditions, the changes of main drilling parameters such as the field bit weight, speed, torque and drilling fluid displacement, will affect the drill string vibration. The effect of speed is more obvious, but the bit weight has quite a small effect and it can restrain drill string vibration by increasing bit weight while in normal drilling.

\section{CONCLUSION AND SUGGESTIONS}

This article proposes a designing scheme of the storable down-hole drilling string vibration testing system. From the system hardware design and software design, our study elaborates the design process of this system.

By the dynamic test of drill string vibration while drilling, the field technicians can comprehend and analyze the underground condition based on the underground vibration signals.

The storable down-hole drilling string vibration testing system has the advantages of small volume, low power dissipation, vibration resistance and high temperature resistance, and it can adapt to the drilling field test.

When the data transmission technology is mature, the vibration data synchronous transmission and storage technology can be considered for the demand of real-time production guidance.

\section{CONFLICT OF INTEREST}

The authors confirm that this article content has no conflict of interest.

\section{ACKNOWLEDGEMENTS}

This work is supported by scientific research starting project of SWPU (No. 2014QHZ029), national natural science foundation of China (No. 51504211) the State administration of national security (No. sichuan-009-2013 AQ, No. sichuang-0021-2014 AQ), and the Sichuan educational committee (No. 15ZB0060).

\section{REFERENCES}

[1] D. C. Chen, B. Comeaux, and G. Gillespie, "Real-Time Downhole Torsional Vibration Monitor for Improving Tool Performance and Bit Design", IADC/SPE Drilling Conference, 2006.

[2] A. Goke, P. Julio, and P.Benny, "Implementation of a Shock and Vibration Mitigation Process: Achieving Real-Time Solutions and Savings", SPE Drilling \& Completion, vol. 24, no. 02, pp. 301-310, 2009.

[3] P. Flavio, and M. Francesco, "Seismic while drilling fundamentals of drill-bit seismic for exploration," Handbook of Geophysical Exploration, 2005.

[4] P. Flavio, M.C. Jose, and L. Massimo, "Acoustic velocity of seismic-while-drilling (SWD) borehole guided waves," Geophysics, vol. 67, pp. 921-927, 2002.

[5] Flavio Poletto. "Energy balance of a drill-bit seismic source, part 1: Rotary energy and radiation properties", Geophysics, vol. 70, no. 2, pp. T13-T28, 2005.

[6] Mark Proett, Mike Walker, David Welshans, et al., "Formation testing while drilling, a new Era information testing", SPE Annual Technical Conference and Exhibition, 2003.

[7] Gravem Trond, "Second Generation of LWD Formation Pressure Testing Technology Improves Data Quality, Increases Sealing Efficiency and Shortens Test Time", SPWLA $47^{\text {th }}$ Annual Logging Symposium, 2006.

[8] G.Heisig et al., "Lateral Drill String Vibrations in ExtendedReach Wells", IADC/SPE Drilling Conference, 2000.

[9] Junsheng Cheng, Dcjie Yu, Jiashi Tang, et al., "Application of frequency family separation method based upon EMD and local Hilbert energy spectrum method to gear fault diagnosis", Mechanism and Machine Theory, 2008.

[10] Bing Li, Pei-lin Zhang, Dong-sheng Liu, et al. "Classification of time-frequency representations based on two-direction 2DLDA for gear fault diagnosis", Applied Soft Computing, vol. 11, no. 8, pp. 5299-5305, 2011.

Received: July 6, 2014

Revised: October 8, 2014

Accepted: December 8, 2014

(C) Ge et al.; Licensee Bentham Open.

This is an open access article licensed under the terms of the (https://creativecommons.org/licenses/by/4.0/legalcode), which permits unrestricted, noncommercial use, distribution and reproduction in any medium, provided the work is properly cited. 